\title{
IROS 2020 - Innovationen in Salzburg
}

Nach dem Jubiläumskongress 2019 luden die Deutsche, Österreichische und Schweizerische Gesellschaft für Interventionelle Radiologie in diesem Jahr, vom 16 . bis 18. Januar 2020, wieder nach Salzburg zur traditionellen 3-Ländertagung ein. Der IROS, der erste Kongress des Jahres für das breite Spektrum interventioneller Themen, ist mittlerweile die größte deutschsprachige Veranstaltung ihrer Art und erfreut sich weiter steigenden Zulaufs. Federführend für ein 9-köpfiges Programmkomitee waren in diesem Jahr Prof. Patrick Knüsel (Chur) und Prof. Andreas Mahnken (Marburg). In besonderer Weise war dieser IROS 2020 Prof. Friedrich Olbert (Wien), dem im April 2019 verstorbenen Gründer des Interventionell-Radiologischen Olbert-Symposiums, gewidmet. Ein Gedenkposter erinnerte in der Eingangshalle zu den Vortragssälen an ihn und seine Verdienste, Errungenschaften und Leistungen für die interventionelle Radiologie.

Thematisch umfasste das Programm, wie in den vergangenen Jahren, nahezu alle Bereiche der interventionellen Radiologie, mit Schwerpunkten in der Gefäßmedizin am ersten und der interventionellen Onkologie am zweiten Kongresstag. Dabei blieb der IROS dem Vorgehen früherer Jahre treu, die Präsentationsformen stetig anzupassen. Neben den klassischen Frontalvortragssitzungen kann das Format „Life-in-Box-Präsentation“ bereits im zweiten Jahr als etabliert gelten. Ein Videomitschnitt eines aufgezeichneten interventionellen Eingriffs wird unter der Moderation des im Saal anwesenden Operateurs und eines weiteren Experten präsentiert und diskutiert. Eine echte Bereicherung, die auch in den engen Grenzen einer 100-Minuten-Sitzung die Abhandlung einer komplexen Prozedur erlaubt. Besonders gut angenommen wurden in diesem Jahr die erstmals ins Pro- gramm integrierten Sitzungen „Brandneues bei vaskulären Interventionen “ und „Brandneues in der Interventionellen Onkologie“. Dabei konnten jeweils in 50-Minuten-Sitzungen je 3 echte Hot-Topics des betreffenden Spezialbereichs beleuchtet werden. Hands-on-Workshops und Simulatorkurse erfreuten sich auch beim IROS 2020 großer Beliebtheit, eröffnen sie doch weniger erfahrenen Anwendern das Kennenlernen auch komplexer Prozeduren unter Laborbedingungen. Das lockt zunehmend auch jüngere Kolleginnen und Kollegen an und ist inzwischen ein wichtiger Baustein sowohl innerhalb der Weiterbildung als auch zur Erhaltung des Fachgebiets. Abgerundet wurde das ärztliche Programm durch die Modulkurse A und C inklusive Prüfungen. Erfreulicherweise ist der Zuspruch der MTRAs bzw. RTs weiterhin sehr hoch, was die Richtigkeit des Grundkonzeptes des Kongresses - eine Kombination aus wissenschaftlicher Theorie und praktischer Anwendung - erneut unterstreicht.

Die deutliche Straffung der Eröffnungsveranstaltung am ersten Kongresstag bei trotzdem ausreichend Zeit für die Überreichung von Ehrenmitgliedschaften und weiteren Auszeichnungen der DeGIR und der ÖGIR hat dem Programm sicher gutgetan. Auch die Integration der „Honorary Lecture“ in die Eröffnungsveranstaltung ist eindeutig ein Gewinn. Prof. Jaschke (Innsbruck) stellte dabei den Strahlenschutz in der interventionellen Radiologie, ein seit Jahren von ihm bearbeitetes Gebiet, in den Mittelpunkt. Auch wenn im Vergleich zu den Anfängen der Radiologie erhebliche Verbesserungen zum Schutz von Patienten und Personal zur Verfügung stehen, ist die Sensibilität für das Thema ständig wach zu halten. Dies auch vor dem Hintergrund, dass kardiologische Bildgebung und Inter-

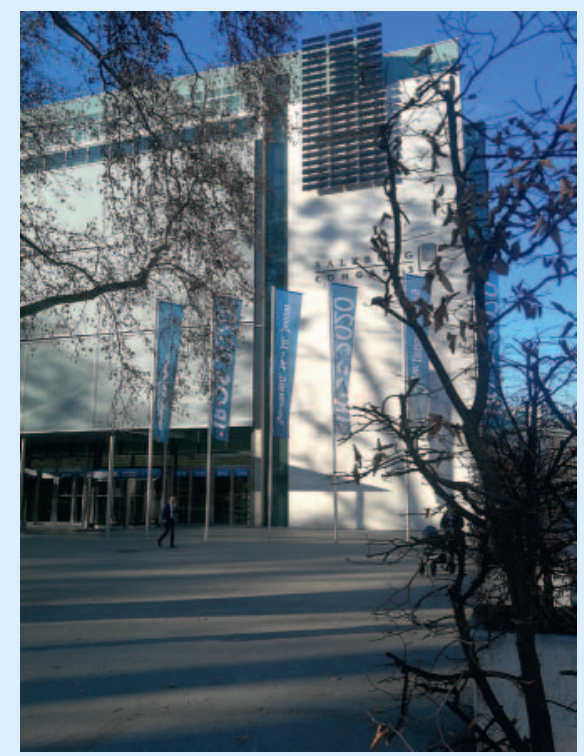

vention zahlenmäßig die beim IROS diskutierten Eingriffe um ein Vielfaches übersteigen. Dennoch haben die verbesserten technischen Möglichkeiten der Angiografie- und CT-Anlagen aufgrund der optimierten Auflösung und zusätzlicher Optionen wie Cone-Beam-CT oder CT-Durchleuchtung manche Eingriffe überhaupt erst möglich gemacht. Dem Anwender muss allerdings immer bewusst sein, dass er hier mit den strahlenintensivsten Verfahren unseres Faches hantiert. Nicht nur die neue europäische Strahlenschutzgesetzgebung, die in der EU unterschiedlich weit umgesetzt ist, hat hierzu deutlich engere Grenzen gezogen. Prof. Jaschke ermahnte alle Anwender, Operateure und Assistenten, zum eigenen Wohl die notwendigen Schutzmaßnahmen zu nutzen und mögliche Dosisreduktionen immer wieder zu hinterfragen. Der interventionelle Radiologe müsse den Strahlenschutz als seine Kernkompetenz verstehen und dabei auch innovativ und konsequent vorangehen. Die Mittel dazu hat er zur Hand. 
Im Themenkomplex „Vaskuläre Interventionen“ wurden neben der klassischen peripheren Rekanalisation auch die an Bedeutung gewinnenden Eingriffe bei zentraler Lungenembolie und Interventionen am Pfortadersystem diskutiert. Dabei stehen Wiedereröffnungen der jeweiligen Kreislaufsysteme zunehmend im Mittelpunkt der Betrachtung. Während die Behandlung der schweren zentralen Lungenembolie als Ultima Ratio, nicht zuletzt wegen der notwendigen zusätzlichen Logistik vor allem in universitären Zentren, in größerem Stil etabliert ist, kommt Interventionen an der Pfortader vor allem im Rahmen der zunehmenden Leberzentren eine immer wichtigere Rolle zu. Dennoch ist für eine qualitativ hochwertige Patientenversorgung ein Angebot in der Fläche für beide Indikationsgruppen anzustreben.

Nach wie vor besteht erhebliche Verunsicherung in der Anwendung medikamentenbeschichteter Ballone und Stents. Auch wenn seit dem Erscheinen der Publikation von Katsanos et al. im Dezember 2018 zahlreiche Veröffentlichungen die damals geäußerten Kernaussagen infrage stellen, bleiben für den Anwender die Empfehlungen der Aufsichtsbehörden und die Verpflichtung zur äußerst restriktiven Anwendung. Weitere angekündigte Publikationen im Jahr 2020 lassen hoffen, dass zumindest eine Klärung und klare Empfehlungen für den Anwender erreicht werden können.

Der Thementag zur interventionellen Onkologie stellte die Behandlung der Lungenmetastase, die Bedeutung ihrer Behandlung durch perkutane Therapieverfahren und die Notwendigkeit einer zur Operation vergleichbaren R0-Situation erneut in den Mittelpunkt. Navigierungssysteme spielen dabei eine immer wichtigere Rolle, insbesondere bei kleinen Herden, deren räumli- che Ausdehnung durch konventionelle Bildkontrolle häufig unterschätzt wird.

Von besonderer Bedeutung werden in der onkologischen Therapie in naher Zukunft neue Therapieformen, einschließlich der Möglichkeiten der intraarteriellen Immuntherapie, sein. Aktuelle Studien lassen hier erhebliche Verbesserungen des therapeutischen Erfolgs erwarten. Die interdisziplinäre Zusammenarbeit von Pathologen, Onkologen, Immunologen, Radiologen und Nuklearmedizinern einschließlich ihrer Medizinphysiker wird dabei wichtiger denn je sein.

Neben den Schwerpunkten setzten Sitzungen zu interventionellen Eingriffen am Lymphsystem, zur interventionellen Endokrinologie, zur Refluxbehandlung im männlichen und weiblichen Becken oder zu den Interventionen rund um den Dialyse-Shunt thematische Akzente. Abgerundet wurde das Programm durch eine „Heiße Eisen“Sitzung zur Analgosedierung und ein Seminar zur Statistik in der interventionellen Radiologie.

Erneut zogen die Sitzungen „Ein Fall, der mich nicht schlafen ließ“, jeweils als Abschluss der ersten beiden Kongresstage, und die „M\&M-Konferenz“ zum Ende des Kongresses am Samstag sehr viele Teilnehmer an. Durch die eingebauten TED-Fragen mit Abstimmungsmöglichkeit über die Kongress-App konnte jeder Teilnehmer die eigene Entscheidung für sich ausloten und in den Gesamtzusammenhang einordnen. Auch das in allen Sitzungen des Kongresses mögliche Stellen von Fragen über die Kongress-App an die Referenten führte zu einer lebhafteren Diskussion bei gleichzeitig straffem und diszipliniertem Zeitmanagement der Sitzungsleiter.
Die an allen Kongresstagen geöffnete Industrieausstellung im Foyer rundete auch den IROS 2020 in bewährter Weise ab. Viele der heute möglichen Therapieverfahren sind erst im engen Austausch zwischen Industrieunternehmen und einzelnen Anwendern entstanden. Umgekehrt bietet der Standbesuch in den Pausen die Möglichkeit, sich über Neu- und Weiterentwicklungen sämtlicher Sektoren interventioneller Maßnahmen zu informieren.

Die Teilnehmer verlassen den IROS 2020 in der Gewissheit, einen Überblick über die aktuellen Themen der interventionellen Radiologie bekommen zu haben, Anregungen für eine Weiterentwicklung der eigenen Abteilung erhalten und im Gespräch mit Kollegen die persönlichen Kenntnisse und Erfahrungen ausgetauscht und erweitert zu haben. Als nächste Möglichkeit zur Standortbestimmung in der interventionellen Radiologie bietet sich 2020 vor allem die in München stattfindende Jahrestagung der CIRSE im Herbst an, bevor der IROS 2021, aus organisatorischen Gründen wieder in Salzburg, zum Jahresauftakt seine Pforten öffnet. Eine starke interventionelle Radiologie wird auch weiterhin neben der Diagnostik das zweite wichtige Standbein zum Erhalt und Ausbau unseres Faches sein.

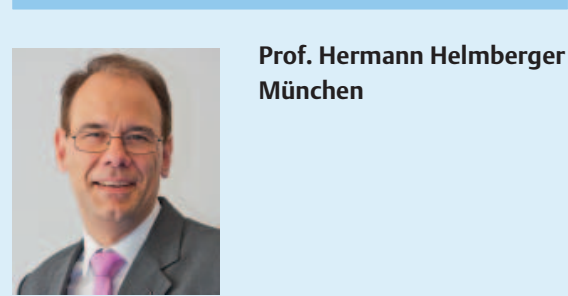

